\title{
Unified Charging and Billing Solution. Unified - Next Generation of Charging Systems in Mobile Networks
}

\author{
Daniel Donhefner \\ Nokia Siemens Networks \\ Business Support Systems Research and Development
}

\section{Motivation and Overview}

The mobile market evolves from commodity voice and simple messaging services to value-added data and multimedia services. This not only implies to move from pure telecom to IT/IP- environment, but to exploit their markets with innovative and differentiated offerings to keep the churn rate low and attract new customers. Communication Service Providers (CSP) must focus increasingly on meeting individual needs and higher expectations of their subscribers. They expect service packages that can be tailored to meet the specific demands of their personal situation, preferences and lifestyle. This requires a flexible customer-centric approach instead of the legacy historical grown and diversed system architecture and organizations of CSPs.

The CSPs are facing the challenge to set up a complete operational environment in a very short time frame. However this is also the opportunity to reach a truly future proof and modern solution and gain competitiveness if the optimal architectural decisions are made now. Nokia Siemens Networks offers a full turnkey solution based on a modern architecture and world market leading products.

The success factors for CSPs are:

- prepaid-postpaid convergence to offer attractive end use services and streamline processes

- solid CRM strategy to improve customer insight and customer loyalty by customer centricity

- state of the art IT architecture via middleware approach

- focus on productized services (rather than project specific solutions) to decrease time to market and reduce OPEX

- stepwise approach to start with basic functions and the option to extend the products in the future evolution

With this Nokia Siemens Networks addresses all these issues and thus considerably extends the scope of competing solutions on the market that only introduce convergence.

A convergent charging and rating platform bundled with value-added applications is an elemental part to fulfill all market requirements. CSP`s marketing teams need flexible tools to enable their efforts for creating new business and defend the existing customer base. Many CSPs worldwide are currently developing their own convergent strategy to be clearly ahead of their competitors that are limited with their traditional 
legacy environment. The method of paying for services is not any longer a reason to separate offers and customers.

Nokia Siemens Networks Unified Charging and Billing solution allows CSPs to overcome the traditional restrictions when shaping innovative and differentiated services that target different customer types, payment methods or network types.

\section{Success factor: Operational efficiency Overcoming business silos}

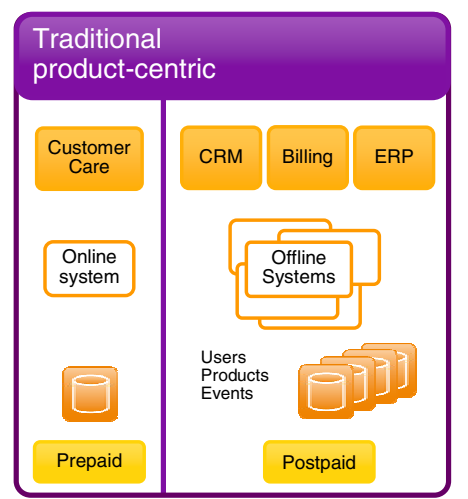

- Limited offerings

- Fragmented environment and processes

- Fragmented Organization / competences
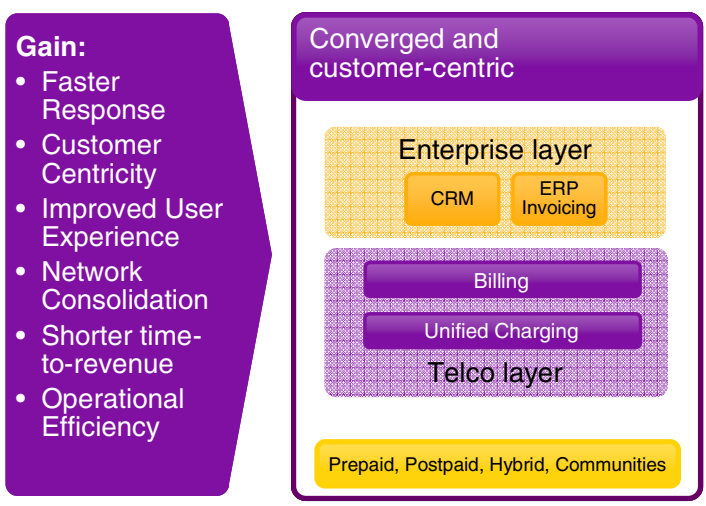

- Flexible targeted offerings

- Customer-centric business processes

- Optimized organization / competences

Fig. 1. Success factor: Operational efficiency - overcoming business silos

Traditionally CSPs had to update their legacy billing systems to cope with every new service. This often leads to a multiplicity of incompatible systems and databases, enlarging the complexity of the IT environment at the CSP. The reward for reducing the number of systems and databases and the simplification of the service introduction process represents the significant reduction in operational costs.

Consequently, simply segmenting customers into prepaid and postpaid is no longer valid. Offering attractive services and stimulating service usage is currently coupled with mastering a multitude of workflows and legacy interdependencies. It is not economically justifiable to have two completely separate systems for users who simply have different payment methods.

Shifting to a customer-centric approach brings significant competitive advantages to CSPs in terms of improved business flexibility and faster time-to-market of new services. It optimizes operational efficiency and reduces costs, due to reduced number of systems and interfaces, and using synergies of formerly separated pre- and postpaid systems and organizational structures.

Due to consolidated online information on subscriber's service usage and context, it enables the CSP to create attractive cross-bundling and cross-promotion offers 
across the whole subscriber basis. It enables CSPs to offer the same functionality, services, customer care and cost monitoring to all their customers, regardless, if they pay in advance before using the services, or if they will be invoiced on a regular basis after usage. With the possibility of having a complete customer insight across all service usage, offers can be made even more personal and targeted. Offering all combinations of voice, data and content services with prepaid and postpaid pricing plans creates loyalty, attracts new subscribers and can be used to promote higher revenue generating services like data.

\section{Unified 2-layer architecture Charging and Billing \& Customer Care and Financials}

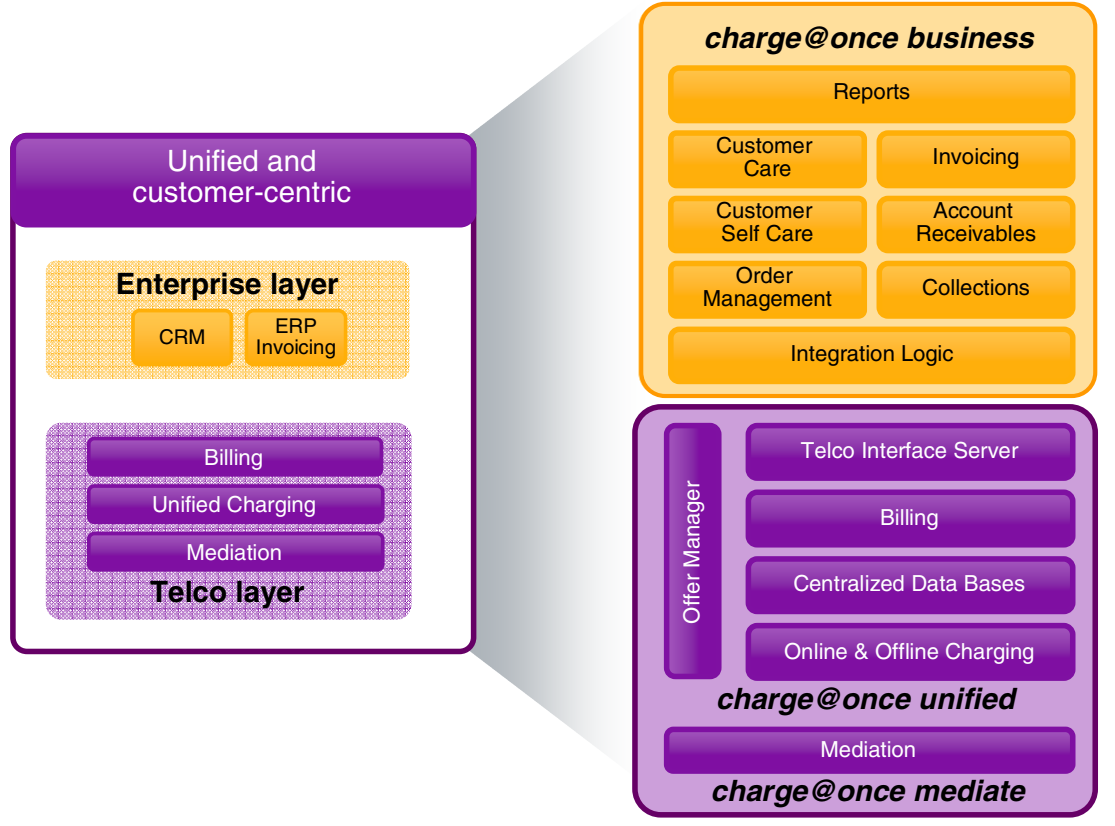

Fig. 2. Unified 2-layer architecture - Charging and Billing \& Customer Care and Financials

Instant, online charging information is a vital tool for stimulating service usage. One of the major factors inhibiting the uptake of new value-added services is the concern of customers that they may be committing themselves to open-ended expenditure. In an end user research carried out by Nokia Siemens Networks end of 2006, more than $80 \%$ of respondents said, that knowing the cost of a service in advance would make them much more likely to use it. Furthermore, online charging allows CSPs to make balance-related offers and improves customer interaction. Prepaid customers in the fastest growing markets appreciate the opportunity of always having an up-to-date prepaid balance visible in their phone display. This is an example of the customer-centric features supported by our solutions.

Online charging and credit control implemented to postpaid customers also reduces the financial risks for CSPs. If a subscriber overruns his credit limit by using a voice 
service, the loss to the CSP is small. But new content-based services with higher tariffs significantly increase the financial risk. The exposure is even higher when the service or application is provided in partnership with a content provider who is entitled to a percentage of the amount paid by the user. If the customer fails to pay his bill, then the CSP has not only lost his own margin, but also has to pay the content provider. Online charging reduces the CSP's exposure to such losses.

With unified charging and billing the CSP can now benefit from having online and offline charging in the same solution. The possibility to use the optimum charging method, independent of the payment method means full flexibity for the offered services. At the same time it is possible to maximise the return on investment by choosing the cheaper offline charging method when the online control is not needed.

Making the shift to a customer-centric approach brings significant advantages to CSPs in terms of improved business flexibility, efficiency and reduced operational costs. The added flexibility enables them to introduce new services that will boost revenues and win greater market share - and gives them powerful measures to react to competition without simply having the opportunity to reduce prices.

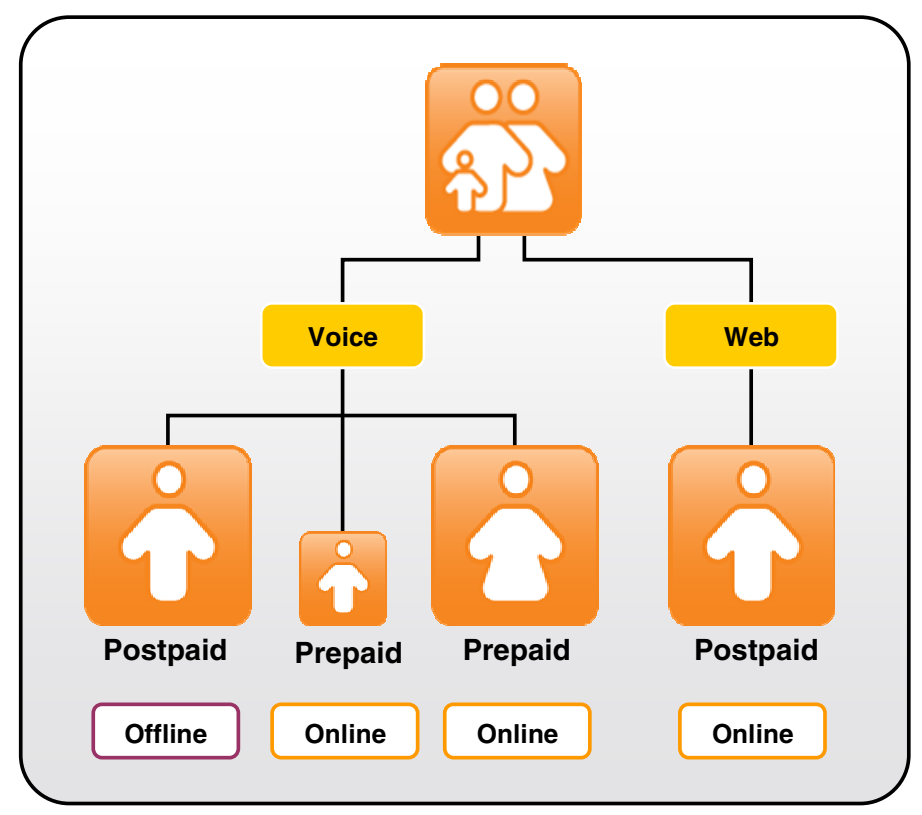

Fig. 3. Customer-centric approach

Nokia Siemens Networks believes to have the best solution for the CSPs for the following reasons:

- Rely on Market Leading Solution Providers. The solution is built on proven and world market leading products. Nokia Siemens Networks provides convergent charging and billing charge@once unified. In addition charge@once business 
consists of rebranded and pre-integrated Oracle products for CRM (Siebel), Invoicing (eBS) and Middleware (SOA Suite).

- Profit from Nokia Siemens Networks End-to-End Commitment and Strong Regional Presence. Nokia Siemens Networks has a strong regional footprint in all regions of the world, including an own system integration branch CSI.

- Enhance Competiveness by Distinguished Productized Approach and Flexible Customization. The productized approach together with high flexibility through onsite adaptation (adapt@once) allows CSP to profit from frequent product enhancements and to quickly introduce new services.

- Go With the Pre-Integrated Two-Layer Architecture, the Future Architectural Standard for BSS. The two layer approach is replacing the traditional billing systems by splitting its functionalities between the Telco specific charging and enterprise generic CRM and ERP including Financials. This allows to deploy best in class products and to gain from economy of scale.

- Put CRM in the Proper Place. Unlike some competitor offerings, which are centered around billing, Nokia Siemens Networks builds on a CRM as the master of the main customer data.

- Gain with a Stepwise Evolution to Reach Your Business Targets with Minimal Risk. Nokia Siemens Networks offers a smooth stepwise evolution. This minimizes risks, addresses the business targets of the CSP in a prioritized order and allows to grow in a flexible way.

\section{Solution Overview}

The Unified Charging and Billing solution offers rating, charging and billing calculation of content, events, sessions in SS7 and IP networks independent of the payment method. It is a modular, highly scalable and flexible solution, offering open interfaces for a smooth integration into the CSP's operational environment. Based on the long-term experience of Nokia Siemens Networks in the real-time prepaid market, it provides superior connectivity, scalability, capacity and performance as well as the carrier-grade availability needed for business-critical online charging solutions.

This solution is an evolutionary step towards a two-layer architecture work split between a transaction-latency optimized "Telco Layer" and an "Enterprise Layer" which is telecom agnostic and dedicated to fast enterprise process integration. With this horizontally layered architecture every function applies to the entire customer base and to the entire product portfolio - overcoming the historical grown diversity of systems and processes between pre- and postpaid and resulting vertical borders for fast, flexible and consistent product shaping and customer segmentation.

As a CSP, you win flexibility, data consistency and reduce your OPEX, credit risks and revenue leakage due to the consolidation of system environment and business processes.

This approach simplifies and accelerates operational workflows and puts CSPs into the position to focus on fast roll-out of any offering to any member of the customer base i.e. to offer all services, product bundles, and quality of services to all customer and access types. 


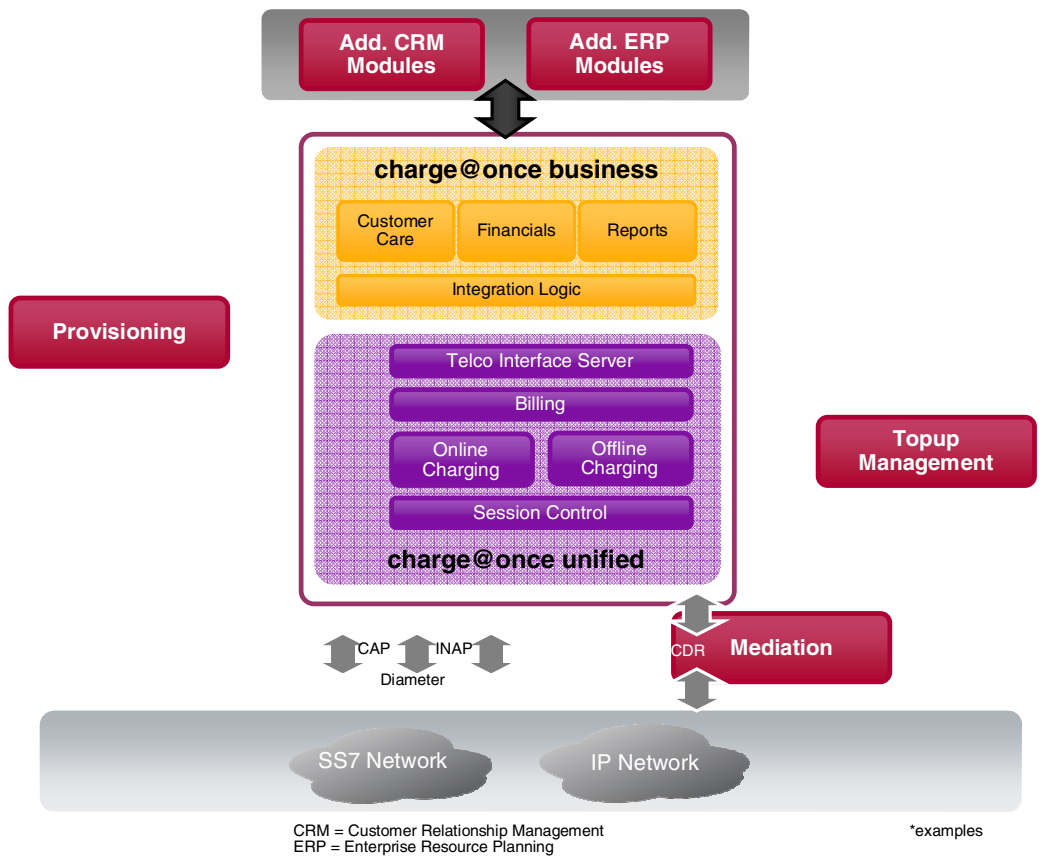

Fig. 4. Unified Charging and Billing - modular architecture

\section{Two-Layer architecture}

\section{Entire customer base with a single horizontal layer}

Enterprise Layer holds tasks common across all types of industries

- Customer Relationship Management (CRM) and customer care

- Enterprise Resource Planning (ERP), payments and collections

- Invoicing, account receivables, bill presentation and archiving

- Off-the-shelf enterprise application

- State-of-the-art enterprise application integration

Telco Layer holds tasks specific to telecommunication industry

- Multiple network access

- Online (highest performance and reliability) / offline rating and charging, balance management

- Event history and aggregation up to invoice line items

Fig. 5. Two-Layer architecture - entire customer base with a single horizontal layer

The Nokia Siemens Networks Unified Charging and Billing Solution supports all of these scenarios and enables CSPs to create attractive bundles of services and charge for these efficiently, regardless of whether they were designed for a prepaid or 
postpaid subscriber. This will allow CSPs to differentiate themselves in the market place, thus driving up the ARPU and increase loyalty of subscribers.

\section{Benefits at a Glance}

The optimized architecture for all applications, all networks and all users enables fast adaptation to market needs, and significantly reduces the operational cost of managing multiple systems and workflows for Prepaid and Postpaid customers. The Unified Charging and Billing solution allows to eliminate legacy billing systems and covers the different availability requirements for online and offline processing with dedicated functional components in a cost-optimized way.

For a CSP, the evolution to a Unified Charging and Billing solution offers following benefits:

- CSPs can rapidly deliver differentiated and targeted solutions to meet market and individual customer demands and thus increase their service revenues

- Introduction of services regardless of payment method of the subscriber

- The ability to deliver attractive pricing schemes, increased service selection to all customers, and an improved personalized care and self-care will attract new customers and maximize the retention of existing customers.

- Online charging, cost control and price notification services that help end-users to keep control of their spending will actually make them confident to use valueadded services, and above all it reduces the entry barriers for new offerings

- Financial risks for the CSP can also be minimized as all services can be charged in advance or supervised in real time and customers can easily be moved to credit supervision and vice versa.

- Online or near-online rating and charging for all new and innovative applications as well as for traditional services in mobile, fixed, SS7 and IP networks prevents from revenue leakages.

- A consolidated system generates significant reductions in operational costs through reduced complexity, improved data management and reduced correction efforts.

- The solution is designed around a complete set of CSP business process descriptions using an end-to-end view for customer interaction, product definition and billing/charging.

- Integration is simplified due to use of same logical data definition across the solution, the open APIs and the powerful Telco Interface Server ensure the seamless integration with Enterprise Layer components and external systems as well - be it a financial system, CRM, Inventory management system or any other commercial off-the shelf system.

- Efficient new hardware reduces OPEX due to less floor-space, increased calculation performance and less energy consumption 